\title{
UCRL-CONF-237013
}

LAWRENCE LIVERM ORE NATIONAL LABORATORY

\section{Dielectric function of warm dense gold}

Y. Ping, D. Hanson, I. Koslow, T. Ogitsu, D.

Prendergast, E. Schwegler, G. Collins, A. Ng

December 5, 2007

APS/DPP annual conference

Orlando, FL, United States

November 12, 2007 through November 16, 2007 
This document was prepared as an account of work sponsored by an agency of the United States government. Neither the United States government nor Lawrence Livermore National Security, LLC, nor any of their employees makes any warranty, expressed or implied, or assumes any legal liability or responsibility for the accuracy, completeness, or usefulness of any information, apparatus, product, or process disclosed, or represents that its use would not infringe privately owned rights. Reference herein to any specific commercial product, process, or service by trade name, trademark, manufacturer, or otherwise does not necessarily constitute or imply its endorsement, recommendation, or favoring by the United States government or Lawrence Livermore National Security, LLC. The views and opinions of authors expressed herein do not necessarily state or reflect those of the United States government or Lawrence Livermore National Security, LLC, and shall not be used for advertising or product endorsement purposes. 


\title{
Dielectric function of warm dense gold
}

\author{
Y. Ping ${ }^{1}$, D. Hanson ${ }^{2}$, I. Koslow ${ }^{2}$, T. Ogitsu ${ }^{1}$, D. Prendergast ${ }^{3}$, E. Schwegler ${ }^{1}$, G. Collins ${ }^{1}$, and $^{\text {A. Ng }}{ }^{1}{ }^{2}$ \\ ${ }^{1}$ Lawrence Livermore National Laboratory, Livermore, CA, U.S.A. \\ ${ }^{2}$ Department of Physics \& Astronomy, University of British Columbia, Vancouver, B.C., Canada \\ ${ }^{3}$ Lawrence Berkeley National Laboratory, Berkeley, CA, U.S.A.
}

(Dated: November 30, 2007)

\begin{abstract}
We review single-state measurements of the broadband (450-800 $\mathrm{nm}$ ) dielectric function of gold with a supercontinuum probe, which have demonstrated the first evidence of the existence of band structure in ultrathin gold foils isochorically heated by a femtosecond laser pulse to energy densities of $10^{6}-10^{7} \mathrm{~J} / \mathrm{kg}$. The Drude component of the dielectric function increases with energy density while the inter-band component shows both enhancement and red shift. Ab initio molecular dynamics calculations based on thermalized electrons can not reproduce the experimental results, suggesting a non-Fermi distribution of excited electrons.
\end{abstract}

PACS numbers: 52.50.Jm, 52.25.Fi, 52.25.Os, 52.38.- $-\mathrm{r}$

\section{INTRODUCTION}

Warm dense matter lies in a regime where densities are near the solid density and temperatures are between 0.1 and $100 \mathrm{eV}$. These warm and dense states are characterized by comparable thermal and Fermi energies of electrons and strongly correlated ionic cores [1] . At the crossroad between condensed matter physics and plasma physics, the behaviors of warm dense matter are very difficult to model because they involve degenerate, stronglycoupled states which evolve through non-perturbative, non-adiabatic processes. On the other hand, the highenergy-density nature of warm dense matter makes experimental study also challenging. Data obtained from expanding systems are integrated over mixed states, thus difficult to interpret. This has left warm dense matter a largely uncharted frontier. Nonetheless, warm dense matter is drawing increasing attention because of its role in understanding the convergence between condensed matter and plasma physics, as well as its relevance to other areas including shock physics [2], inertial confinement fusion [3] and astrophysics [4]. Among this broad interest, a new focus is non-equilibrium warm dense matter. This is driven by its practical importance as transient states in laboratory-produced high energy density matter and its fundamental significance in the study of relaxation processes and phase transitions.

A key parameter characterizing warm dense matter is its dielectric function, $\varepsilon(\omega)$. Encompassing contributions from intra-band and inter-band transitions, it is central to the understanding of electron transport and optical properties. This can be seen in the imaginary part of dielectric function, $\varepsilon_{2}(\omega)$, of solid and liquid gold [5-7], as plotted in Fig. 1 for photon energy $E_{p h}=0.5-5.0 \mathrm{eV}$. The $\varepsilon_{2}(\omega)$ at the solid phase shows two pronouced features: a Drude-like profile from free-electron contributions for $E_{p h}=0.5-2.0 \mathrm{eV}$, and a "bump" above $2.0 \mathrm{eV}$ which is due to $d-p$ transition in gold. In contrast, the $\varepsilon_{2}(\omega)$ at the liquid phase is just a monotonic decreasing function of photon energy. Therefore, the dielectric function, especially broadband $\varepsilon_{2}(\omega)$ in a photon energy range covering

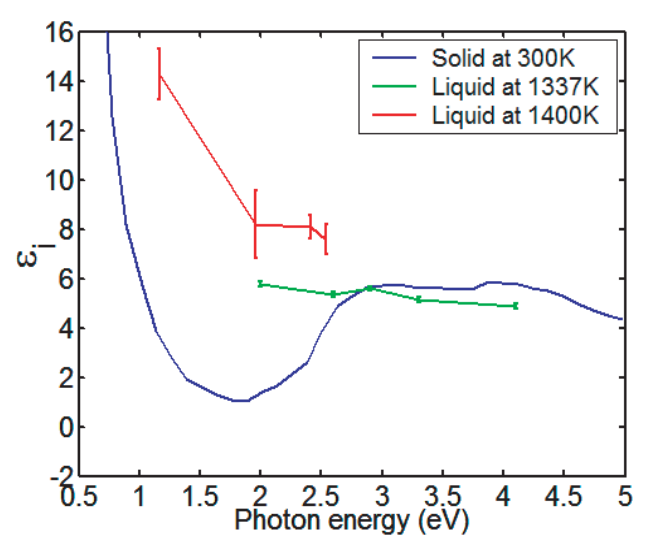

FIG. 1: The imaginary part of dielectric function of solid and liquid gold for a photon energy range of $0.5-5.0 \mathrm{eV}$. The data are from Ref. [5-7].

interband transition regions, is a manifestation of band structure and density of state effects, thus rendering it a plausible means of probing structural phases.

It is of intense interest to the study of warm dense matter produced not only by laser excitation but also by shock compression. Changes in $\varepsilon_{2}(\omega)$ in low energy density states have been studied extensively in thermomodulation spectroscopy [8-15] including reflectivity and transmissivity measurements. The only available singlestate data on the dielectric properties of highly nonequilibrium warm dense matter are the $\mathrm{DC}$ and $\mathrm{AC}$ conductivities of gold heated by a femtosecond laser to energy densities of $10^{5}-10^{7} \mathrm{~J} / \mathrm{kg}$ [16]. However, the latter is measured at a single photon energy of $1.55 \mathrm{eV}$ (wavelength of $800 \mathrm{~nm}$ ) and is thus the result of intra-band transitions. On the other hand, ab initio calculation of the dielectric function of aluminum across the solidliquid transition has been obtained [17]. Most recently, AC conductivity of warm dense gold in a spectral range that covers both intra-band and inter-band transitions has been reported albeit based on very limited Brillouin zone sampling [18]. 
In this paper we review the experimental techniques and results of the broadband measurements, which yields the first single-state data at high energy densities with simultaneous observations of intra-band and inter-band components with sub-ps resolution [19]. The results verify Drude-like behavior at $800 \mathrm{~nm}$. They also reveal an enhancement and red-shift of the $d-p$ band transitions that persist until the heated sample disassembles. The measured data provide a critical benchmark for theory and point to the importance of adequate Brillouin zone sampling to ensure convergence in calculations. The $a b$ initio dielectric function with thermal electron distribution, i.e., the Fermi distribution function, does not reproduce the interband transition peak above $2.3 \mathrm{eV}$, suggesting a non-equilibrium distribution of excited electrons.

\section{EXPERIMENTAL METHOD}

The experiment is performed on the Europa Laser at LLNL using a pump-probe technique (femtosecond laser pump and supercontinuum probe [20]). The targets are free-standing gold nanofoils (25-33 nm thick) with central flatness better than $\lambda / 10$ over a $300-\mu \mathrm{m}$ region as determined with a $632.8-\mathrm{nm}$ Michelson interferometer. As shown in Fig. 2(a), a 150-fs FWHM, frequency-doubled laser pulse provides the pump for isochoric heating of the gold foils. The 400-nm pump pulse is focused onto the nanofoil at normal incidence with a spot diameter of 80 $\mu \mathrm{m}$ (FWHM). The incident, reflected and transmitted pump light are monitored by both calibrated photodiodes with integrating spheres and CCD cameras to yield measurements of laser deposition across the focal spot with a spatial resolution of $5 \mu \mathrm{m}$. While the skin-depth of 400-nm light is only $\sim 7 \mathrm{~nm}$ in gold, the electron ballistic range is $\sim 110 \mathrm{~nm}[21]$ that far exceeds the thickness of the target. Combined with the 150 -fs pulse width, this results in uniform and isochoric heating of the nanofoil at solid density and allows the determination of excitation energy density $\rho_{E}$ directly from the laser deposition measurement. The energy density cited in this paper is the average over the central $15-\mu \mathrm{m}$ region where the profile of the pump pulse is almost flat.

The supercontinuum probe is generated by focusing a 180-fs FWHM, 800-nm pulse onto a $\mathrm{CaF}_{2}$ crystal [22]. A typical supercontinuum spectrum is shown in Fig. 2(b), which extends from 1.50 to $2.85 \mathrm{eV}$. It is line-focused onto the gold nanofoil at a $45^{\circ}$-incidence, covering both heated and unheated regions of the target as shown in Fig. 3(a). The unheated region provides in-situ spectral calibration since the room-temperature dielectric function of gold is well known. The spatially-resolved reflected and transmitted spectra, recorded with two intensified CCD cameras, are displayed in Fig. 3(b). The ratio of reflection/transmission in the heated central $15-\mu \mathrm{m}$ region to the unheated region is obtained directly from the spectra after background subtraction. The heated reflectivity $\mathrm{R}^{*}$ and transmissivity $\mathrm{T}^{*}$ are then calculated by multiplying

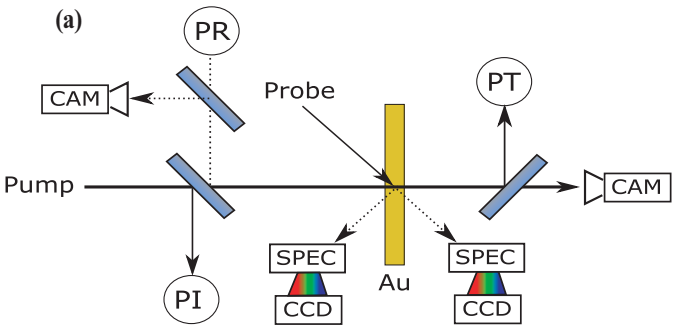

(b)

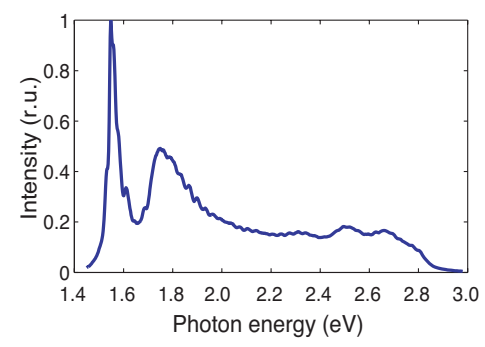

FIG. 2: (a) Experimental schematic. PI, PR and PT are photodiodes for measuring input energy, reflected energy and transmitted energy of the pump pulse. (b) Supercontinuum spectrum.

(a)

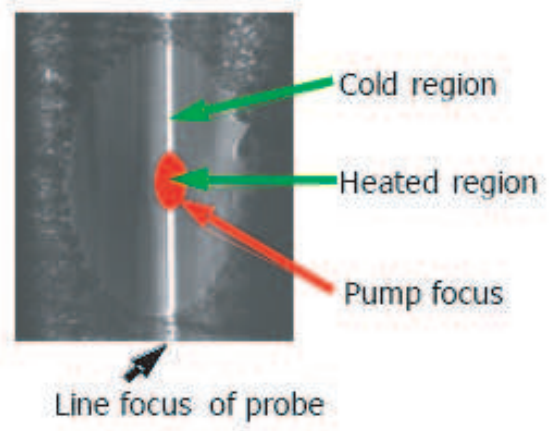

(b)

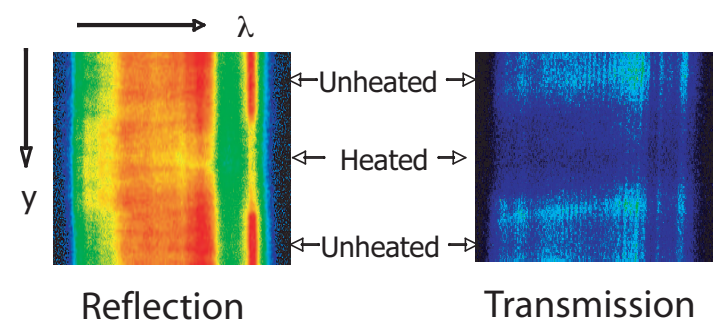

FIG. 3: (a) Image of the target with a line focus of the supercontinuum probe. (b) Spatially resolved reflected and transmitted spectra.

the ratio with the values at the room-temperature for a known target thickness.

The conversion from $\mathrm{R}^{*}$ and $\mathrm{T}^{*}$ to dielectric function $\varepsilon(\omega)$ is carried out by numerically reversing the Fresnel formulae for a gradient-free dielectric slab [23] in accordance with the Idealized Slab Plasma concept [24]. An 

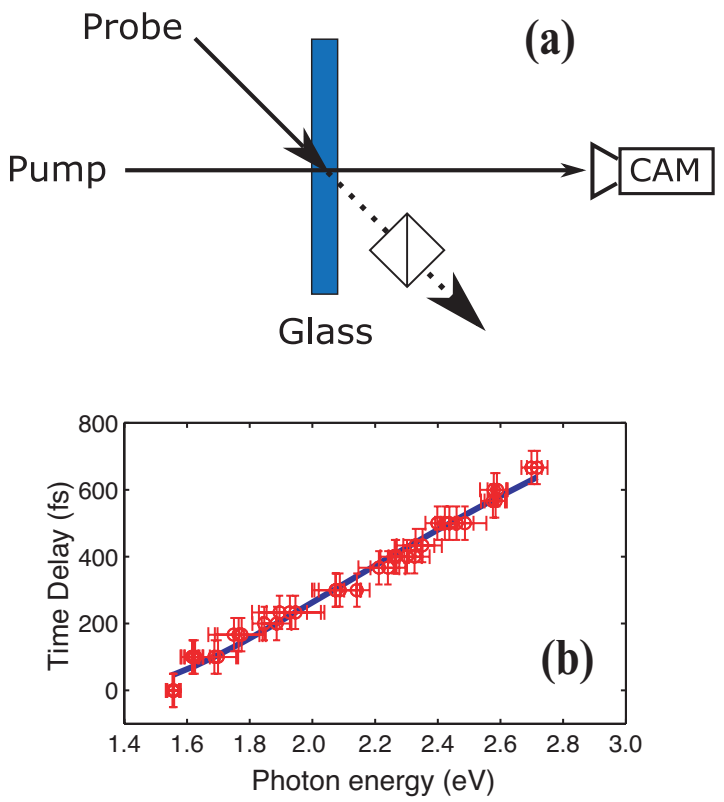

FIG. 4: (a) Setup for chirp measurement. (b) Frequency chirp in the supercontinuum probe.

elegent algorithm by Paulick [25] is implemented to solve for the complex refractive index $\hat{n}=n+i k$ instead of the dielectric constant. The two are equivalent, related by $\varepsilon=\left(\varepsilon_{1}+i \varepsilon_{2}\right)=(n+i k)^{2}=n^{2}(1+i \kappa)^{2}=\hat{n}^{2}$. The use of $\hat{n}$ enables us to take advantage of the physical meaning of $k / n=\kappa$ as an 'attenuation index', determining the exponential decay of the probe wave as it propagates through the foil. For fixed $n, \mathrm{~T}^{*}$ decreases monotonically with increasing $k$. The monotonicity allows us to reduce the $2 \mathrm{D}$ problem of locating $\left(\varepsilon_{1}, \varepsilon_{2}\right)$ to two $1 \mathrm{D}$ problems, which can be solved more accurately and efficiently. In the case of multiple solutions, we use the rule of thumb that for noble metals the solution having the lowest real part of the refractive index $\hat{n}=\sqrt{\varepsilon}$ is the physical one [26]. In the case that a true solution is not found (due to measurement errors in $\mathrm{R}^{*}$ and $\mathrm{T}^{*}$ ), we accept approximate solutions [27] only if the percentage discrepancy with the measured $\mathrm{R}^{*}$ and $\mathrm{T}^{*}$ values is less than $10 \%$. The data are averaged over 10 shots to further reduce the measurement errors. The final error bars in $\left(\varepsilon_{1}, \varepsilon_{2}\right)$ are shown in Figs. 5 and 6.

This broadband probe transmits through optics before reaching the target, therefore a frequency chirp is introduced in the temporal profile. The chirp is measured using the Kerr optical-gate technique [28] and the setup is shown in Fig. 4(a). The Au target is replaced by a $100 \mu \mathrm{m}$-thick glass. A polarizer is added in exiting path of the S-polarized probe to minimize the transmission of probe. The heating pulse is employed as the gating pulse to induce nonlinear effects in the glass, which rotates the polarization of the probe. As a result, part of the probe pulse that temporally overlaps with the gating pulse inside the glass leaks through the crossing polarizer and appears as a bright spot in the spectrum. By scan- ning the delay betwwen the gating pulse and the probe, the temporal profile of the supercontinuum is obtained as shown in Fig. 4(b). To remove the effect of chirp in time-resolved measurements, spectral data are binned in 10-nm intervals and appropriate temporal shifts are applied using Fig. 4(b).

\section{EXPERIMENTAL RESULTS AND DISCUSSION}

The temporal evolution of $\varepsilon(\omega)$ of gold at an excitation energy density of $(2.9 \pm 0.3) \times 10^{6} \mathrm{~J} / \mathrm{kg}$ is shown in Fig. 5 . The data have been corrected for the frequency chirp as described above. Time zero corresponds to the on-set of changes in $\mathrm{R}^{*}$ and $\mathrm{T}^{*}$ from their room temperature values. This is found to be the same for all frequencies in the supercontinuum spectrum after chirp correction. Also included in the figure are tabulated data of gold at room temperature [5]. The time span is chosen to cover an initial transient followed by a quasi-steady state as discovered in our earlier studies [16, 29]. At a photon energy of $1.55 \mathrm{eV}$, the dielectric function showed an initial transient consisting of a decrease (increase) in the real (imaginary) part to a minimum (maximum) value at $\sim$ $0.8 \mathrm{ps}$, then followed by an increase (decrease) to a quasisteady-state value at $\sim 1.2 \mathrm{ps}$. This is consistent with our previous phase shift measurements [29]. A single $\varepsilon(\omega)$ plot is presented for $1.2-4.0 \mathrm{ps}$ since no significant change in $\varepsilon(\omega)$ is observed in the interval, which confirms the existence of a quasi-steady state for the observed spectral range.

From photon energies $1.55 \mathrm{eV}$ to $2.60 \mathrm{eV}, \varepsilon_{1}(\omega)$ appears relatively featureless. However, intra-band and inter-band $(d-p)$ components are clearly discernable in $\varepsilon_{2}(\omega)$ below and above $\sim 2.3 \mathrm{eV}$. They both show substantial enhancements over their room-temperature values. Furthermore, by displaying $\varepsilon_{2}(\omega)$ at different time steps with an offset (Fig. 5(c)), it can readily be seen that the intra-band component shows good agreement with best-fitted Drude functions [30] that assume frequencyindependent electron collision time and density, except possibly for a small region around $1.6 \mathrm{eV}$ at the peak of the transient at $800 \mathrm{fs}$. The electron collision time and density deduced from the Drude fitting are listed in Table. 1.

To examine the dependence of $\varepsilon(\omega)$ on the excitation energy density $\rho_{E}$, we use measurements made on the quasi-steady state. The results are presented in Fig. 6 . These are not corrected for frequency chirp and the probe delay varies from $1.4 \mathrm{ps}$ at $1.55 \mathrm{eV}$ to $2.0 \mathrm{ps}$ at $2.6 \mathrm{eV}$. Time zero again corresponds to the onset of observed changes in $\mathrm{R}^{*}, \mathrm{~T}^{*}$ at $1.55 \mathrm{eV}$. For $\rho_{E}$ of $2.2 \times 10^{6}$ and $4.7 \times 10^{6} \mathrm{~J} / \mathrm{kg}$, the $1.4-2.0$ ps probe delay falls completely within the quasi-steady state duration [29] allowing the dielectric function to be determined over the entire spectral range of $1.5-2.7 \mathrm{eV}$. However, at $1.7 \times 10^{7} \mathrm{~J} / \mathrm{kg}$ no accepatable solution can be found for $\varepsilon(\omega)$ from the $\mathrm{R}^{*}$, 


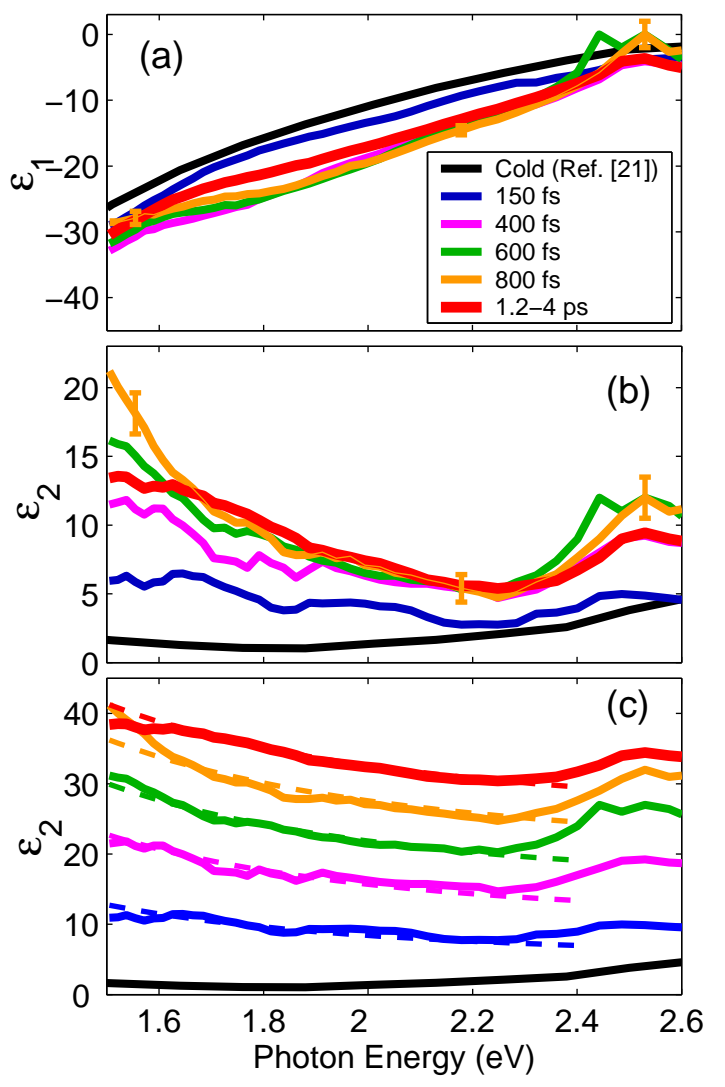

FIG. 5: (a) $\varepsilon_{1}(\omega)$ and (b) $\varepsilon_{2}(\omega)$ at different times for an energy density of $2.9 \times 10^{6} \mathrm{~J} / \mathrm{kg}$, and (c) $\varepsilon_{2}(\omega)$ displayed with an off-set of +5 along the $y$-axis between time steps. Sample error bars are included in (a) and (b). The dashed curves are best-fit Drude functions.

$\mathrm{T}^{*}$ data above $2.38 \mathrm{eV}$. This is due to the breakdown of the uniform slab assumption as target disassembly gives rise to gradients in the expanding foil. The cut-off point at $2.38 \mathrm{eV}$ corresponds to a probe delay of $1.9 \mathrm{ps}$ that is consistent with the disassembly time observed in the earlier experiment [29]. Accordingly, dielectric function measurement using a frequency chirped source offers a new means of probing hydrodynamic disassembly of a heated solid.

With increasing excitation energy density, the quasisteady-state $\varepsilon_{1}(\omega)$ begins to reveal the effect of interband $(d-p)$ transitions above $2.1 \mathrm{eV}$ while $\varepsilon_{2}(\omega)$ exhibits increasing enhancements in intra-band and inter-band transitions. The intra-band component continues to be Drude-like as indicated by the best-fit Drude functions in the figure. The fitting parameters are also given in Table 1. It should be noted that the quasi-steady state collision time and electron density are in good agreement with previous single-wavelength measurements at $800 \mathrm{~nm}$ $(1.55 \mathrm{eV})[16]$. The inter-band component also shows a red shift that increases with excitation energy density.

A basic property contained in the spectral dielectric function is electronic joint density of state. Thus, the data in Fig. 5 carry information on the evolution of the

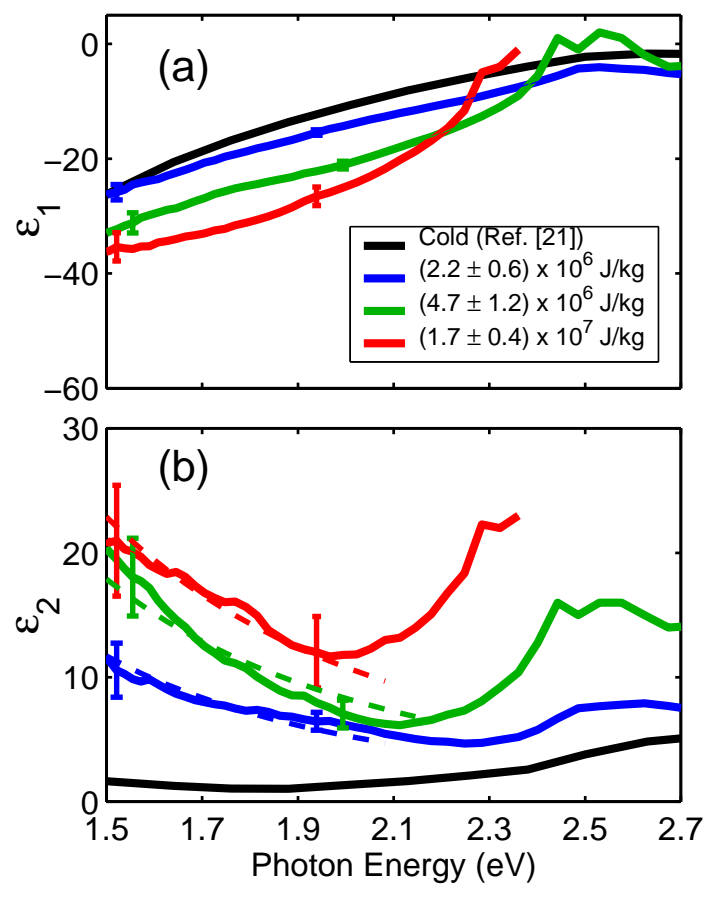

FIG. 6: (a) $\varepsilon_{1}(\omega)$ and (b) $\varepsilon_{2}(\omega)$ for different excitation energy densities. Sample error bars are included. The dashed curves are best-fit Drude functions.

TABLE I: Parameters for Drude fitting in Figs. 5 and 6.

\begin{tabular}{ccccc}
\hline \hline & $\Delta \epsilon\left(10^{6} \mathrm{~J} / \mathrm{kg}\right)$ & Probe Delay & $\tau\left(10^{-15} \mathrm{~s}\right)$ & $\mathrm{n}_{e}\left(10^{22} \mathrm{~cm}^{-3}\right)$ \\
\hline (a) & 2.90 & $150 \mathrm{fs}$ & 1.49 & 4.54 \\
(b) & 2.90 & $400 \mathrm{fs}$ & 1.16 & 6.01 \\
(c) & 2.90 & $600 \mathrm{fs}$ & 0.97 & 6.34 \\
(d) & 2.90 & $800 \mathrm{fs}$ & 0.87 & 6.45 \\
(e) & 2.90 & $1.2-4.0 \mathrm{ps}$ & 0.83 & 5.93 \\
(f) & 2.20 & $1.4-2.0 \mathrm{ps}$ & 1.00 & 5.10 \\
(g) & 4.70 & $1.4-2.0 \mathrm{ps}$ & 0.86 & 7.00 \\
(h) & 17.0 & $1.4-2.0 \mathrm{ps}$ & 0.77 & 8.40 \\
\hline \hline
\end{tabular}

density of the $d$ and $s / p$ states driven by the processes of photo-excitation of $d$-electrons, electron-hole recombination and electron-electron thermalization at high energy densities. Similarly, the data in Fig. 6 carry information on the dependence of density of state on excitation energy density. For the range of conditions of interest, Drude-like behavior of the intra-band component of $\varepsilon_{2}(\omega)$ is confirmed. This provides a crucial validation for the use of AC conductivity at $800 \mathrm{~nm}$ to derive DC conductivity, collision time and carrier density of warm dense gold [16], thus significantly enhancing the utility of AC conductivity measurement.

The appearance of inter-band transitions presents the first evidence of the persistence of $d$-band in the quasisteady state of ultrafast laser heated gold. Based on the theoretical formula for dielectric function, for example, Kubo-Greenwood formalism, a peak in the $\omega$ dependence indicates the existence of a pair of parallel band separated by a near-constant energy. If the system 
becomes disordered, it is expected that the band structure will be disturbed, and the peaks in the dielectric function will be smeared out. Therefore, one could speculate that the observed enhanced inter-band transition peak reflects the presence of long-range ordering of ions, and that the quasi-steady state is the characteristic of a super-heated solid which would be consistent with a recent report based on an empirical two-temperature model [18]. Equally important are the observed increases in red shift and enhancement of $d-p$ transitions with excitation energy density. Red shifts in the inter-band transition region may result from temperature-induced changes in the energy distribution of the electrons. The enhancement is however unexpected. Calculations of optical absorption spectrum of equilibrium solid and liquid phases of aluminum [17] show only red shifts in inter-band transitions but no enhancements as the solid is heated to its melting point, consistent with observation [31]. In order to understand the mechanism for the enhanced interband contribution, we performed first-principle simulations as discussed below.

\section{IV. $A B$ INITIO CALCULATIONS}

The calculation of $\varepsilon_{2}(\omega)$ is based on density functional theory within local density approximation [32]. We approximate the ionic cores of the gold atoms using Troullier-Martins pseudopotentials [33] and we expand the electron wave functions in a plane wave basis using a kinetic energy cut-off of 40 Ry. All the calculations are carried out at the equilibrium density of gold at ambient condition (fcc unit-cell with the lattice constant at $4.052 \AA$ ), since earlier measurements indicate the absence of significant expansion during the quasi-steady state of the gold foil $[16,29] . \varepsilon_{2}(\omega)$ is then calculated using the Kubo-Greenwood formula [34], where only interband contributions are considered and a frozen core is adopted to approximate the all-electron valence wave functions in our estimation of the necessary velocity matrix elements [35]. A crucial aspect of such an ab initio approach is the numerical convergence in integrating the Brillouin zone. This can greatly impact the accuracy in calculating inter-band contributions to the dielectric function [17], especially in the case of gold, where the characteristic transition peaks originate from a small area in the Brillouin zone where branches of the bands involved become parallel. Therefore, we first focus on the assessment on the convergence of the dielectric function with respect to the $\mathrm{k}$-point mesh size to integrate the Brillioun zone.

The calculation results of $\varepsilon_{2}(\omega)$ for a perfect fcc gold crystal at $0 \mathrm{~K}$ are shown in Fig. 7. It is clear that convergence is reached using a uniform grid of $128^{3} k$-points. Our estimate of $\varepsilon_{2}(\omega)$ is in excellent agreement with that obtained from an all-electron localized basis-set approach [36]. This indicates that for a 32-atom gold system, sampling over $65,000 k$-points is required to ensure fidelity

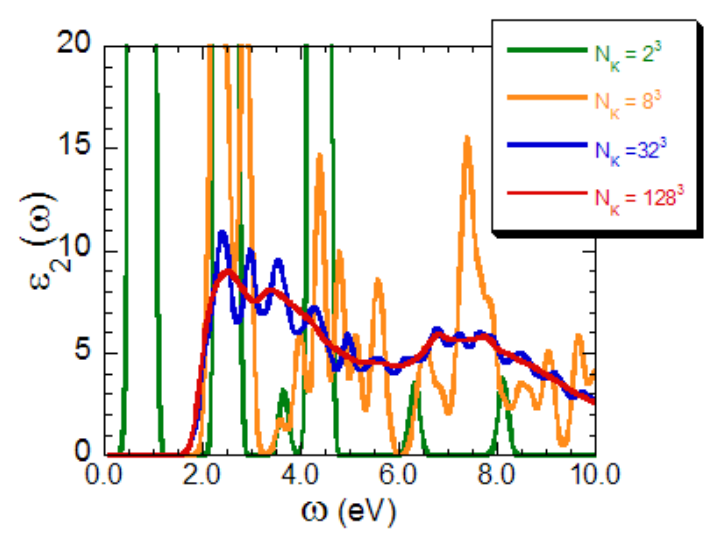

FIG. 7: $\varepsilon_{2}(\omega)$ of gold at $0 \mathrm{~K}$ calculated with different Brillouin zone sampling. $\mathrm{N}_{k}$ is the number of $k$-points.

of the calculation. It is also evident from Fig. 7 that insufficient Brillouin zone sampling can readily generate substantial fluctuations in the dielectric function. This may account for the highly structured conductivities seen in recent calculations of a 32 -atom system with sampling over only $4 k$-points [18], which roughly corresponds to $8^{3} k$-points with a FCC unit cell. It may also contribute to the spurious local maximum seen at $1.7-1.9 \mathrm{eV}$ giving it a non-Drude appearance in Ref. [18], which deviates substantially from our observation described above.

We now turn to the $\varepsilon_{2}(\omega)$ in the experimental condition. The first challenge is the quantum mechanical nature of the evolution of the system. The following is a rough sketch of the dynamics of laser excited material. At time $t=0$, only the electrons (not ions) are excited by the laser pulse, then the excited electron would decay through light radiation, phonon excitation and/or electron-electron scattering. To calculate the time evolution of $\varepsilon_{2}(\omega)$, we need to know both the ionic configuration and the electronic structure for a given time. However, the processes described before are quantum mechanical in nature, meaning that it is given as the solution of the time dependent Schrodinger equation, for which a practical solution does not exist. Therefore, the current approaches to this problem use crude approximations. In the most commonly used approach, the twotemperature model, we assume that both electronic and ionic degree of freedom are in thermal equilibrium conditions individually, but each degree of freedom has its own temperature. The energy dissipation between those two degrees of freedom is described by a single parameter, the electron-phonon coupling constant, which is usually estimated from the one at ambient condition. Generally, considering that the thermal disorder will smear out the structural feature in $\varepsilon_{2}(\omega)$, it will be useful to examine if the low ionic temperature with high electron temperature, which is essentially the condition that the two-temperature model provides, could produce the main feature of the quasi-steady state. The ab initio molecular dynamics at $T_{i o n}=300 \mathrm{~K}$ has been performed with the 


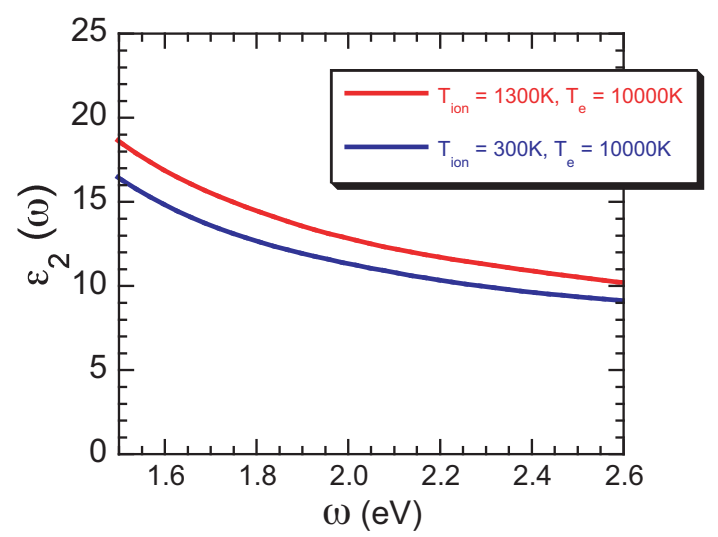

FIG. 8: Calculated $\varepsilon_{2}(\omega)$ of gold at three temperatures.

32-atom supercell, from which a few ionic configurations were selected. The $\varepsilon_{2}(\omega)$ for those configurations were calculated at $T_{e}=10000 K$. For comparison, the $\varepsilon_{2}(\omega)$ with $T_{\text {ion }}=1300 K$ (near the melting temperature) and $T_{e}=10000 K$ was calculated with the same procedure. The results are shown in Fig. 8. Although the absolute values of $\varepsilon_{2}(\omega)$ at $\omega=2.6 \mathrm{eV}$ are in reasonable agreement with experiments, we do not find the enhancement of the inter-band transition peak near $2.5 \mathrm{eV}$ at all, which is in conflict with experimental observation.

At last, we analyze the potential source of the discrepancy. There are a few critical approximations in the theoretical treatment: 1 . The electronic degree of freedom is in thermal equilibrium, i.e., the Fermi-distribution function was used; 2. The Kubo-Greenwood formalism is applied in the Drude regime. Let us first explain the second approximation. The Kubo-Greenwood formalism account only the elastic electron phonon scattering, while in the Drude regime, inelastic scattering is known to play an important role. In our simulation, as we use the supercell, the band folding takes place, which introduces the artificial intra-band scattering without the inelastic electron-phonon scattering. Although the impact is not precisely understood, it is reasonable to conjecture that the Drude part will be overestimated due to the missing scattering channel. Nevertheless, it is hard to conceive that inclusion of the inelastic scattering explain the observed enhancement on the inter-band transition peak; this effect should affect on the lower energy range, below $2.5 \mathrm{eV}$. The first approximation, the Fermi distribution function of electron, might have more crucial impact on the structure of dielectric function. If the electronic temperature is high, the contribution from higher energy bands would increase due to the enhanced occupations of electrons above the Fermi level. Generally, this contribution suppress the peak since a pair of bands with a similar separation in higher energy is not expected. Therefore, one might speculate that an enhancement of a peak in dielectric function might require a non-equilibrium (nonFermi) electronic distribution function. On the other hand, measurements of electron energy distribution function of ultrafast laser excited gold [37] have shown that even at very low excitation energy densities $\left(300 \mu \mathrm{J} / \mathrm{cm}^{2}\right.$ or equivalently $\sim 5 \times 10^{5} \mathrm{~J} / \mathrm{kg}$ for a $30-\mathrm{nm}$-thick gold foil), a small energetic electron tail appears to remain visible 670 fs after the laser pump pulse. We are thus led to conjecture that our observed enhancements in $d-p$ transitions are likely the manifestation of non-equilibrium density of state resulting from the complex processes of electronhole recombination and electron-electron relaxation in states driven to very high energy densities.

\section{CONCLUSION}

In conclusion, our broadband measurements have given us some interesting insights to the behavior of ultrafast laser excited gold. The existence of a quasi-steady state, as observed in single-wavelength measurements, is confirmed for the whole visible region. It is found that the dband contribution in the dielectric function is persistent through the quasi-steady state. This provides the first evidence of band structure survival in non-equilibrium warm dense gold. In addition, our data have provided the first benchmark for testing theory. We believe that insufficient Brillouin zone sampling is the likely cause for the noted discrepancy between the published theoretical calculations [18] and our experiment. This points to the need for examining proper convergence of numerical calculations with regard to finite-size effects. Our measurement have also shown the enhancement of d-band component upon ultrfast laser heating, which can not be reproduced in $a b$ initio calculations of the dielectric function based on thermalized electrons. This might suggest a non-Fermi distribution of excited electrons.

We wish to thank L. Bendict for valuable discussions. This work was performed under the auspices of the U.S. Department of Energy by Lawrence Livermore National Laboratory in part under Contract W-7405-Eng-48 and in part under Contract DE-AC52-07NA27344, and was also supported by the Natural Sciences \& Engineering Research Council of Canada.
[1] S. Ichimaru, Rev. Mod. Phys. 54, 1017 (1982).

[2] A. Ng et al., Laser \& Particle Beams 23, 527 (2005).

[3] T.R. Dittrich et al., Phys. Plasmas 6, 2164 (1999).

[4] D. Saumon et al., High Pressure Res. 16, 331 (2000).
[5] P. B. Johnson and R. W. Christy, Phys. Rev. B 6, 4370 (1972).

[6] J. C. Miller, Philos. Mag. 20, 1115 (1969).

[7] S. Krishnan, G. P. Hansen, R. H. Hauge and J. L. Mar- 
grave, High Temp. Science 29, 17 (1990).

[8] W.J. Scouler, Phy. Rev. Lett. 18, 445 (1967).

[9] R. Rosei and D.W. Lynch, Phys. Rev. B 5, 3883 (1972).

[10] R. Rosei et al., Surface Science 37, 689 (1973).

[11] R. Rosei, Phys. Rev. B 10, 474 (1974).

[12] E. Colavita et al., Phys. Rev. B 20, 4864 (1979).

[13] J.E. Nestrell Jr. et al., Phys. Rev. B 21, 3173 (1980).

[14] E. Colavita et al., Phys. Rev. B 27, 4684 (1983).

[15] H.E. Elsayed-Ali and T. Juhasz, Phys. Rev. B 47, 13599 (1993).

[16] K. Widmann et al., Phys. Rev. Lett. 92, 125002 (2004).

[17] L. X. Benedict, J. E. Klepeis and F. H. Streitz, Phys. Rev. B 71, 064103 (2005).

[18] S. Mazevet et al., Phys. Rev Lett. 95, 085002 (2005).

[19] Y. Ping et al., Phys. Rev Lett. 96, 255003 (2007).

[20] L. Huang, J. P. Callan, E. N. Glezer and E. Mazur, Phys. Rev. Lett. 80, 185 (1998).

[21] J. Hohlfeld et al., Chem. Phys. 251, 237 (2000).

[22] R. Huber, H. Satzger, W. Zinth and J. Wachtveitl, Opt. Commun. 194, 443 (2001).

[23] M. Born and E. Wolf, Principles of Optics, Cambridge University Press, 1980.
[24] A. Forsman et al., Phys. Rev. E 58, R1248 (1998).

[25] T. C. Paulick, Appl. Opt. 25, 562 (1986).

[26] P. O. Nilsson, Appl. Opt. 7, 435 (1968).

[27] P. Gadenne and G. Vuye, J. Phys. E 10, 733 (1977).

[28] C. Nagura et al., Applied Optics 41, 3735 (2002).

[29] T. Ao et al., Phys. Rev. Lett. 96, 055001 (2006).

[30] P. Drude, Ann. Phys. (Leipzig) 1, 566 (1900).

[31] A.G. Mathewson and H.P. Meyers, J. Phys. F: Met. Phys. 2, 403 (1972).

[32] D. M. Cepewrley and B. J. Alder, Phys. Rev. Lett. 45, 556 (1980). J. P. Perdew and A. Zunger, Phys. Rev. B 23, 5048 (1981).

[33] N. Troullier and J. L. Martins, Phys. Rev. B 43, 1993 (1991).

[34] R. Kubo, J. Phys. Soc. Japan 12, 570 (1957). D. A. Greenwood, Proc. Phys. Soc. (London) A71, 585 (1958).

[35] H. Kageshima and K. Shiraishi, Phys. Rev. B 56, 14985 (1997).

[36] P. Romaniello and P. L. de Boeij, J. Chem. Phys. 122, 164303 (2005).

[37] W. S. Fann et al., Phys. Rev. B 46, 13592 (1992). 